\title{
Ground State Phase Properties of the Penson-Kolb-Hubbard Model with Attractive Pair-Hopping Interaction
}

\author{
W.R. CzART* AND S. RobaszKIEWICZ \\ Electron States of Solids Division, Faculty of Physics, Adam Mickiewicz University, \\ Umultowska 85, 61-614 Poznań, Poland
}

\begin{abstract}
We study the ground state phase diagrams of the extended Hubbard model with the pair-hopping interaction $J$, i.e. the Penson-Kolb-Hubbard model. We concentrate on the case of attractive $J(J>0)$ which can stabilise $s$-wave pairing superconductivity in the system and competes with the on-site repulsive $U$. The evolution of the phase diagrams and order parameters for arbitrary electron density and representative values of the interaction parameters are analyzed within the (broken symmetry) Hartree-Fock approximation. The calculations are performed for the case of nearest-neighbors electron hopping $t$ on the $d=2$ square lattice.
\end{abstract}

DOI: $10.12693 /$ APhysPolA.127.278

PACS: 74.20.-z, 71.28.+d, 74.20.Mn

\section{Introduction}

The extended Hubbard model with pair hopping interaction i.e. the so-called Penson-Kolb-Hubbard (PKH) model is conceptually simple effective model for studying correlations and electron orderings and for description of superconductivity in narrow band systems with shortrange, almost unretarded pairing [1-12]. The model Hamiltonian is the following:

$$
\begin{aligned}
H & =-t \sum_{\langle i j\rangle \sigma}\left(c_{i \sigma}^{+} c_{j \sigma}+\text { H.c. }\right)-\sum_{i \sigma} \mu n_{i \sigma}+U \sum_{i} n_{i \uparrow} n_{i \downarrow} \\
& -\frac{1}{2} J \sum_{\langle i j\rangle}\left(c_{i \uparrow}^{+} c_{i \downarrow}^{+} c_{j \downarrow} c_{j \uparrow}+\text { H.c. }\right),
\end{aligned}
$$

where $t$ is the single electron hopping integral, $U$ is the onsite density-density interaction, $J$ is the pair hopping (intersite charge exchange) interaction, $\mu$ is the chemical potential, the limit $\langle i j\rangle$ restricts the sum to nearest neighbors.

In this report we present the ground state phase diagrams and order parameters evolution of the PKH model for the case of attractive $J(J>0)$ which favorizes $s$ wave pairing state, i.e. the state with the Cooper-pair center-of-mass momentum $\boldsymbol{q}=0$ and which competes with repulsive $U$. The properties of the model will be compared with those of the attractive Hubbard $(\mathrm{AH})$ model $[13,14]$. The calculations are performed for arbitrary electron density $(0<n<2)$ within the (broken symmetry) Hartree-Fock approximation. The case of repulsive pair hopping interaction $(J<0)$ which can stabilize eta-pairing superconductivity and, for $U<0$, charge ordering will be discussed in a separate work [15].

We determine a set of self-consistent equations as the minimum of the free energy of the system $F$ with respect

\footnotetext{
${ }^{*}$ corresponding author; e-mail: czart@amu.edu.pl
}

to the variational parameters: order parameters of considered orderings $x_{\alpha}(\alpha=\mathrm{S}, \mathrm{F}, \mathrm{AF})$ and chemical potential $\mu$, i.e. by the equations

$$
\frac{\partial F}{\partial x_{\alpha}}=0 \text { and } \frac{\partial F}{\partial \mu}=0
$$

where superconducting order parameter for $s$-wave pairing $x_{s}=\frac{1}{N} \sum_{i}\left\langle c_{i \downarrow} c_{i \uparrow}\right\rangle$, antiferromagnetic with the staggered magnetization order parameter $x_{\mathrm{AF}}=$ $\frac{1}{2 N} \sum_{i, \sigma} \sigma \exp \left(\boldsymbol{Q}_{i} \cdot \boldsymbol{R}_{i}\right)\left\langle c_{i \sigma}^{+} c_{i \sigma}\right\rangle,\left(\boldsymbol{Q}=\frac{\Pi}{a}, \frac{\Pi}{a}, \ldots\right)$, and ferromagnetic order parameter $x_{\mathrm{F}}=\frac{1}{N} \sum_{i, \sigma} \sigma\left\langle c_{i \sigma}^{+} c_{i \sigma}\right\rangle$.

We have studied the case of alternating (hypercubic) lattices. We have restricted our analysis to the one- and two-sublattice site orderings and considered only homogeneous phases S, F, AF. In order to determine the phase boundaries we have compared the corresponding free energies of the phases. The paper does not consider mixed ordered phases, bond orderings and phase separated states, which are now under investigation.

\section{Results and discussion}

We have analysed the ground state properties of the model (1) for $d=2$ square (SQ) lattice. In the following we present numerical results for the case restricted to the nearest-neighbors single electron hopping $t$ and pair hopping $J$. We determine the mutual stability of $s$-wave pairing superconductivity (S), ferromagnetic $(\mathrm{F})$, antiferromagnetic $(\mathrm{AF})$ and nonordered $(\mathrm{N})$ phases in such a case.

The ground state diagrams and ordering parameters evolution for various representative values of the system parameters are shown in Figs. 1-3.

In Figs. 1, 2 the phase diagrams are plotted as a function of interactions $(U, J)$ and a concentration $n$, whereas Fig. 3 shows the plots of order parameters as a function of the concentration $n$ and the on-site interaction $U$ for fixed values of the other model parameters.

The diagram $U / 4 t$ vs. $n$ for $J / 4 t=1$ is given in Fig. 1 . All the phase transitions lines between ferromagnetic, 


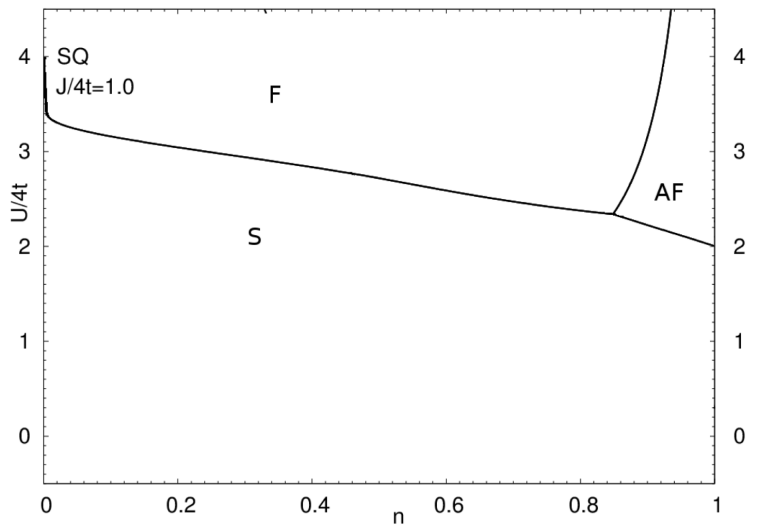

Fig. 1. The phase diagram as a function of $U$ and $n$ for fixed $J / 4 t=1$. All the phase transition lines are of the first-order. Denotations: ferromagnetism (F), antiferromagnetism (AF) and $s$-wave superconducting state $(\mathrm{S})$. All diagrams are plotted for nonfrustrated 2D square lattice (SQ).

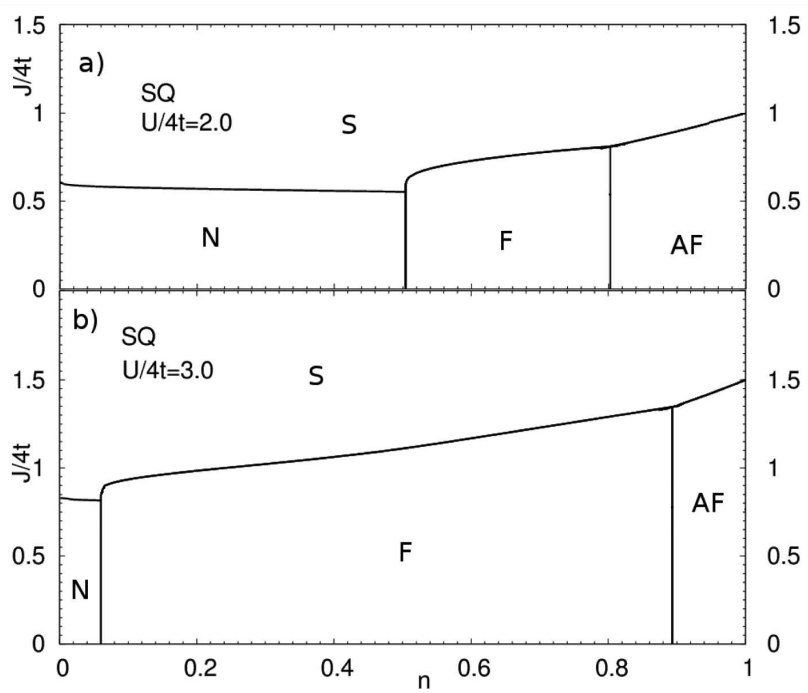

Fig. 2. The phase diagrams as a function of $J$ and $n$ plotted for: (a) $U / 4 t=2$ and (b) $U / 4 t=3$. The transitions between ordered phases are of the first-order and those involving nonordered (N) phase are of the secondorder. Other denotations as in Fig. 1.

antiferromagnetic and $s$-wave superconducting orderings are of the first order. Ferromagnetic and antiferromagnetic phases appear on the diagram above a critical value of repulsive $U\left(U_{\mathrm{c}}\right)$ dependent on $n$, whereas $s$-wave superconducting state is stable for $U<U_{\mathrm{c}}$. In the diagram $\mathrm{AF}$ phase occurs in a finite range of concentration $n$ close to half-filling.

The ground state phase diagrams $J$ vs $n$ for two fixed values of $U$ are shown in Fig. 2. The transitions between ordered phases are of first order while the transitions to nonordered phase are of second order. As the repulsive on-site interaction $U>0$ competes with $J>0$
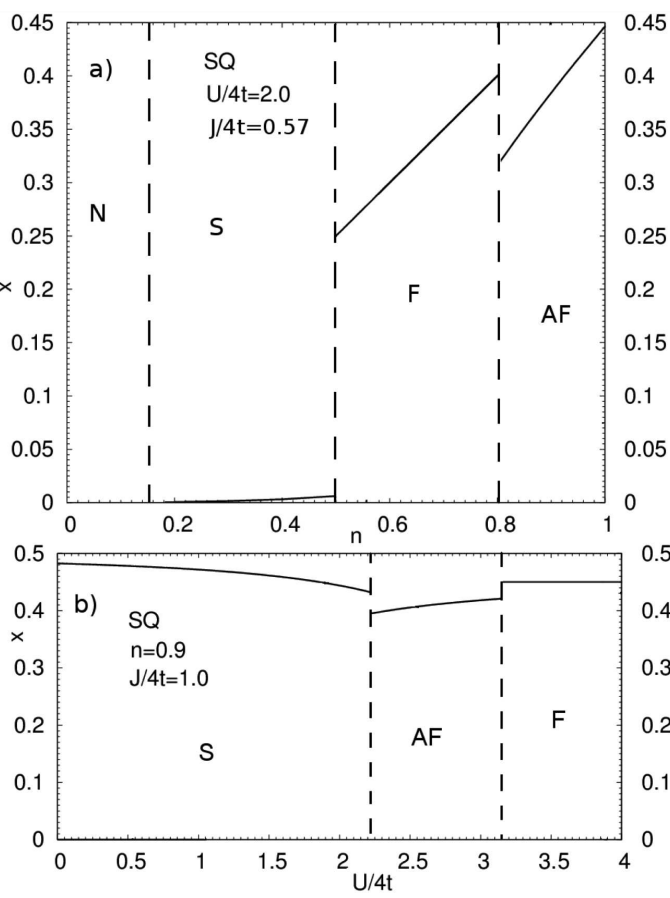

Fig. 3. Order parameters (a) as a function of carriers concentration $n$ for fixed values of interaction parameters $U / 4 t=2.0$ and $J / 4 t=0.57$, (b) as a function of $U$ for fixed values of parameters $n=0.9$ and $J / 4 t=1.0$. The stability ranges of the different phases are indicated by the vertical lines. Symbol $x$ denotes order parameter $\left(x_{s}, x_{\mathrm{F}}\right.$ and $\left.x_{\mathrm{AF}}\right)$ for respective phases. Other denotations as on Figs 1, 2.

the $\mathrm{S}$ phase appears only above some critical value $J / 4 t$, which depends on $U / 4 t$ and $n$. Increase of $U / 4 t$ broadens the range of concentrations $n$ for which the ferromagnetic state is stable and reduces the ranges of antiferromagnetic and nonordered phases (compare Fig. 2a and b).

Our analysis of a general case of arbitrary $U / 4 t$ and $J / 4 t$ shows that for $J>U / 2$ the $\mathrm{S}$ phase is the lowest energy state at $T=0$ for any values of single electron hopping and carrier concentration $(0<n<2)$.

Examples of the concentration dependence of the order parameters for the fixed values of interactions and their evolution with increasing $U$ for fixed $n$ and $J / 4 t$ are presented in Fig. 3a and b, respectively. At the points of the first-order transitions between ordered phases the values of the order parameters are finite, whereas superconducting order parameter vanishes $\left(x_{s} \rightarrow 0\right)$ at the second-order transition to nonordered phase (compare Fig. 3a with Fig. 2a) and at the empty-band limit $n=0$. For strong interactions nonordered phase disappears from the phase diagram (see Fig. 3b and Fig. 1). In Fig. 3b one can see the evolution of the order parameters passing through a sequence of first-order phase transitions (S-AF-F) with increasing $U$ (compare Fig. 1).

Comparing the properties of the superconducting $s$ wave phase of the attractive Hubbard model $(U<0$, 
$J=0$ ) with those of the PKH model with intersite attractive pair hopping interaction $J>0$ for $U=0$ (i.e. the PK model) one finds in both cases gradual evolution from the BCS-like limit to the tightly bound pairs regime with increasing couplings $[1,3,13,14]$. In both cases the interactions stabilize the $s$-wave superconductivity for any coupling strength. However the nonlocal pairing mechanism (intersite charge exchange) makes the dynamics of electron pairs in the PK model to be substantially different from that in the $\mathrm{AH}$ model. As a result these two systems present, except for the weak coupling limit, different thermodynamic and electrodynamic properties behaviours $[1,3,13,14,16]$.

In the $\mathrm{AH}$ model with increasing $|U|$ the inverse square London penetration depth $\lambda^{-2}(T=0)$ continuously decreases, while the $T_{\mathrm{c}}$ increases exponentially for small $|U|$, then it goes through a round maximum and decreases as $t^{2} /|U|$ for large coupling (cf. e.g. Fig. 10 in Ref. [3]). Analogous behavior as $T_{\mathrm{c}}$ exhibits the thermodynamic critical field $H_{\mathrm{c}}(0)$ (cf. e.g. Fig. 5 in Ref. [3]).

On the other hand, in the PK model the $\lambda^{-2}(0)$ increases with $J$, there is no maximum of $T_{\mathrm{c}}$ and $H_{\mathrm{c}}^{2}(0)$ at the intermediate $J$ and both these quantities increase linearly with $J$ for large coupling (Refs. [1, 3, 16]).

As we have seen repulsive $U$ restricts the range of stability of S-phase in the PKH model and can generate either first or second order transitions to various nonsuperconducting states. Moreover it can modify the properties of S-phase [7, 17].

More detailed study of the model, considering also possible mixed orderings and phase separations and analyzing the influence of repulsive $U$ on thermodynamic and electromagnetic properties of S-phase for $d$-dimensional hypercubic lattices will be given elsewhere [17].

\section{Acknowledgments}

We thank P. Grzybowski, K. Kapcia and R. Micnas for helpful discussions.

\section{References}

[1] S. Robaszkiewicz, B. Bułka, Phys. Rev. B 59, 6430 (1999) and references therein.
[2] G.I. Japaridze, E. Muller-Hartmann, J. Phys. Condens. Matter 9, 10509 (1997); G.I. Japaridze, A.P. Kampf, M. Sekania, P. Kakashvili, Ph. Brune, Phys. Rev. B 65, 014518 (2001).

[3] W. Czart, S. Robaszkiewicz, Phys. Rev. B 64, 104511 (2001); A. Ptok, D. Crivelli, K.J. Kapcia, Supercond. Sci. Technol. 28, 045010 (2015).

[4] S. Robaszkiewicz, W. Czart, Acta Phys. Pol. B 32, 3267 (2001)

[5] W.R. Czart, S. Robaszkiewicz, Acta Phys. Pol. A 106, 709 (2004); A. Ptok, K.J. Kapcia, arXiv:1502.00579.

[6] M. Mierzejewski, M. Maśka, Phys. Rev. B 69, 054502 (2004); A. Ptok, M. Maśka, M. Mierzejewski, J. Phys. Condens. Matter 21, 295601 (2009).

[7] S. Robaszkiewicz, W. Czart, Phys. Status Solidi B 236, 416 (2003).

[8] W.R. Czart, S. Robaszkiewicz, B. Tobijaszewska, Phys. Status Solidi B 244, 2327 (2007).

[9] K. Kapcia, S. Robaszkiewicz, R. Micnas, J. Phys. Condens. Matter 24, 215601 (2012).

[10] K. Kapcia, Acta Phys. Pol. A 121, 733 (2012); K. Kapcia, J. Supercond. Nov. Magn. 26, 2647 (2013); K.J. Kapcia, J. Supercond. Novel Magn. in press (2015); DOI:10.1007/s10948-014-2906-4 .

[11] K. Kapcia, J. Supercond. Nov. Magn. 27, 913 (2014).

[12] K. Kapcia, S. Robaszkiewicz, J. Phys. Condens. Matter 25, 065603 (2013); K.J. Kapcia, Acta Phys. Pol. A 126, A-53 (2014).

[13] W. Czart, T. Kostyrko, S. Robaszkiewicz, Physica $C$ 272, 51 (1996).

[14] B. Bułka, S. Robaszkiewicz, Phys. Rev. B 54, 13138 (1996); M. Bąk, R. Micnas, J. Phys. Condens. Matter 10, 9029 (1998).

[15] W.R. Czart, S. Robaszkiewicz, Acta Phys. Pol. A 127, 275 (2015).

[16] W. Czart, S. Robaszkiewicz, Acta Phys. Pol. A 100, 885 (2001); W.R. Czart, S. Robaszkiewicz, Acta Phys. Pol. A 97, 217 (2000).

[17] W.R. Czart, S. Robaszkiewicz, in preparation. 